Philosophical Perspectives, 26, Philosophy of Mind, 2012

\title{
PERCEPTUAL PHENOMENOLOGY
}

\author{
Bence Nanay \\ University of Antwerp and Cambridge University
}

\section{Introduction}

I am looking at an apple. The apple has a lot of properties and some, but not all, of these are part of my phenomenology at this moment: I am aware of these properties. And some, but not all, of these properties that I am aware of are part of my perceptual (or sensory) phenomenology. If I am attending to the apple's color, this property will be part of my perceptual phenomenology. The property of being a granny smith apple from Chile is unlikely to be part of my perceptual phenomenology.

Here are two problems for anyone who is interested in conscious experience in general, and perceptual experience in particular:

(a) How can we tell which properties are part of our phenomenology and which ones are not?

(b) How can we tell which properties are part of our perceptual phenomenology and which ones are part of our non-perceptual phenomenology?

I will focus on (b) in this paper. My aim is twofold: I propose a methodology for answering the question of which properties are part of our perceptual phenomenology and I provide an example for how this methodology could be applied.

\section{The Methodology of Contrast Cases}

Both (a) and (b) have been widely discussed (Siegel 2006, Masrour 2011, Pitt 2004, Robinson 2005, Bayne and Montague 2011) and it is universally assumed that the right methodology for deciding how to answer (a) and (b) is by appealing to contrast cases (see Masrour 2011, Siegel 2007, Kriegel 2007 and Bayne 2009 on the methodology of settling these questions). In the case of (a), this means that if we find two token experiences, E1 and E2, that only 
differ in that property $\mathrm{P}$ is represented in E1 but not in E2 and if E1 and E2 are phenomenologically different, then property $\mathrm{P}$ is part of our phenomenology. Similarly, in the case of (b), if we find two token experiences that only differ in that property $\mathrm{P}$ is represented in one but not the other and if the two experiences differ in their perceptual phenomenology, then property $\mathrm{P}$ is part of our perceptual phenomenology.

An example for how this might work in the case of problem (a) above: I am looking at a page written in Arabic script before and after taking an intensive Arabic language class. These experiences are E1 and E2, respectively. My sensory stimulations in E1 and E2 are the same, since I am looking at the very same page, but there is a kind of property that is represented in E2, but not in E1: the property of being meaningful Arabic words (and not merely nicely curving lines). If E1 and E2 differ in their phenomenal character, then this property is part of my phenomenology.

Problem (b): At a dinner party, I'm eating a piece of meat that I take to be chicken, when my host tells me that it is in fact a piece of rat meat (or pigeon, etc; use your favorite disgusting animal). My experience before she told me this is E1; my experience after that is E2. The only difference between E1 and E2 is that there is one property, the property of being a rat, that is represented in E2 but not in E1 - in all other respects, E1 and E2 are the same. If I am really disgusted by rats, then the point can be made that the perceptual phenomenology of E1 and E2 are different: the meat will taste different.

\section{Doing Without Contrast Cases}

The problem with the contrast case methodology for deciding (a) and (b) is that it is difficult to settle disagreements about phenomenology. If I say that E1 and E2 differ in their phenomenal character (or in their perceptual phenomenology) and you deny this, it is not clear how the issue can be decided. This is even more problematic in the case of (b), where intuitions wildly differ with regards to what phenomenal character counts as perceptual. Does the rat meat example really show that the property of being rat meat is part of our perceptual phenomenology? If someone were to claim that this property is part of our non-perceptual phenomenology, it is difficult to see how we could settle this disagreement.

As a result, I propose a methodology for settling (b), at least in the case of some kinds of properties, that does not appeal to contrast cases. The methodology involves close attention to patients with brain lesions.

I take my lead here from Tim Bayne, who used such cases in order to decide (a). He argues that associative agnosia patients lose their ability to recognize objects as belonging to certain sortals (to recognize them as bicycles or stethoscopes) but not their ability to represent the shape, size and color properties of these objects. He concludes that as their phenomenology also 
changes, this change can only be explained with the change in the represented sortal property: hence, sortal properties are part of one's phenomenology (Bayne 2009).

We need to note two aspects of this argument. First, it is an argument about (a) and not about (b): it says nothing about whether sortals are part of our perceptual phenomenology. Second, and more importantly, in spite of Bayne's appeal to patients with brain lesions, the argument still uses the methodology of contrast cases. The patients' experience before the accident that caused the brain lesions is E1 and their experience after is E2. E1 and E2 differ only in that sortals are represented in E1 but not E2. If E1 and E2 differ in their phenomenal character, we can conclude that sortals are part of our phenomenology. But then we encounter the usual problems with the methodology of contrast cases. If one denied that E1 and E2 really differ in terms of their phenomenal character, it is not clear how Bayne could respond.

I want to keep Bayne's emphasis on patients with brain lesions but drop his contrast case methodology. My claim is that if we find patients who (a) consciously see object $\mathrm{x}$, (b) experience property $\mathrm{P}$ of $\mathrm{x}$, but (c) do not experience any lower level properties of $\mathrm{x}$, such as shape, size or color, then we have good reason to conclude that property $\mathrm{P}$ is part of our perceptual phenomenology.

Here is why. Suppose, for reductio, that property $\mathrm{P}$ is part of the subjects' non-perceptual phenomenology. What about their perceptual phenomenology then? It follows from the claim for reductio that these patients lack any perceptual phenomenology of x's properties while they consciously see $\mathrm{x}$. The only property they are aware of is property $\mathrm{P}$, but this property is, by supposition, not part of their perceptual phenomenology. In other words, it follows from the claim for reductio that it is possible to consciously see an object and nonetheless lack visual phenomenology altogether.

This is the skeleton of the argument for keeping apart perceptual and non-perceptual phenomenology. I will now use a case study to show how this argument can be fleshed out.

\section{Case Study: Unilateral Neglect and the Experience of Action-Properties}

To give a case study of how this might work, I argue that a property that is even less obviously perceptual than sortal properties is part of our perceptual phenomenology: the property of being suitable to perform an action with (see also Nanay 2011a, forthcoming). I call properties of this kind actionproperties. ${ }^{1}$ My claim is that action-properties are part of our perceptual phenomenology.

The claim I am making is about perceptual phenomenology. It needs to be distinguished from a similar but importantly different question, namely, the question about whether a property is represented in perception (see Nanay 2011a, 2012, forthcoming). Perception can be conscious or unconscious and 
there may be properties that are perceptually represented but nonetheless fail to show up in our perceptual phenomenology. Further, depending on how we think about the relation between perceptual content and perceptual phenomenology, a property may be part of our phenomenology but nonetheless fail to be part of our perceptual content. The question I am addressing in this paper is about perceptual phenomenology, not perceptual content.

As promised, my argument is based on empirical findings about patients with brain malfunction. More specifically, some patients with symptoms of unilateral neglect are slow and sometimes even unable to find objects in the contralateral side of their visual field if they are defined by salient visual properties (such as their color). Yet, they are capable of, and relatively efficient in, finding objects defined by the action they can be used for - by what I called 'action-properties' (Humphreys and Riddoch 2001, Riddoch et al. 1998, esp. p. 678).

This experimental finding satisfies the conditions for the methodology I sketched at the end of the last section. Let us focus on the moment when the patients spot the object in the contralateral side of their visual field (in the course of those experiments where they are trying to identify the objects with the help of their action-properties).

The first thing to note is that the patients are unaware of the shape, size and color properties of the objects presented to them in the contralateral side of their visual field. This is not particularly surprising as it is held to be true of all unilateral neglect patients. In fact, unilateral neglect is often defined as the lack of awareness of visual details in the contralateral side of one's visual field (see Driver and Vuilleumier 2001 and Kinsbourne 2006 for summaries). ${ }^{2}$

Second, these patients do consciously experience the property of what an object can be used for. In fact, what alerted the experimenters to the possibility of this experiment is that the patients noticed and told the experimenters that when performing visual search tasks, they experience what the objects can be used for (see Humphreys and Riddoch 2001, p. 84). To use the terminology of feature-binding (Treisman 1996), at this moment, the action-property is bound to the object, but the shape and color properties are not.

But should we really accept the claim that unilateral neglect patients are aware of action-properties? Wouldn't it be possible that these patients are like blindsight patients: that they are not aware of any properties, but unconsciously represent some of them, which allows them to perform actions (in this case, the action of picking out objects in the contralateral field)? My answer is that unilateral neglect patients are very different from blindsight patients. We do not, of course, have fool-proof evidence that they are aware of action-properties. We never do, not even in the case of healthy human beings - this is the famous problem of other minds. The point is that we have no more reason to doubt that unilateral neglect patients are aware of action-properties than to doubt that a healthy human adult is. Remember that the experimenters were alerted to this phenomenon that they went on to test empirically because a patient told them that it is easier for him to find objects when he thinks about what the objects can 
be used for. Blindsight patients are not in the position to say any such things. The unilateral neglect patient says that he is aware of these properties of the objects and we have no prima facie reasons to think that he is lying - we have as much justification to conclude that he is aware of the action-properties as we ever do.

One may also have a general worry about the reliance of this argument on the subjects' introspective claims about their phenomenology. The grand aim of this paper is to use empirical findings and not introspection for drawing a distinction between perceptual and non-perceptual phenomenology. Nonetheless, I am accepting claims about the subjects' phenomenology without any further empirical support. Am I entitled to do so? My answer is that I am. Remember that the aim is to get clear about the difference between perceptual and nonperceptual phenomenology. And I use the subjects' introspective report in order to establish whether they are having an experience per se rather than whether they are having a perceptual experience. Thus, I do not use the introspective report of their perceptual phenomenology to support my claim about perceptual phenomenology. Rather, I use the introspective report of what they are aware of - perceptually or non-perceptually - to support my claim about perceptual phenomenology.

Now let us see why these experimental findings are relevant for the purposes of keeping perceptual and non-perceptual phenomenology apart.

It is important to clarify what does not follow from these findings. It does not follow that in the course of perceptual processing the representation of shape and color comes after, and is grounded in, the representation of action-properties. What follows from these findings is only that the phenomenology of actionproperties comes before (hence, cannot be based on) the phenomenology of shape and color: the subjects experience action-properties before they experience shape and color properties.

Here we need to keep claims about representation and claims about phenomenology apart (see Nanay 2011b). Unilateral neglect is normally described as a disorder of visual attention. The general idea is that the patients are unaware of the contralateral side of their visual field because they cannot unlock their attention from the other side of their visual field. One reason to think so is the following (Mark et al. 1988). If a unilateral neglect patient is unaware of the left side of her visual field, then in a line crossing task, she will only cross the lines in the right side of her visual field. But if the task is not line crossing, but line erasing, that is, if the visual stimulus is slowly removed from the right side of her visual field, then she will eventually erase all the lines, including the ones on the left side of her visual field.

In other words, I take it to be the consensual view about unilateral neglect that these patients do have some kind of representation of the visual details on the contralateral side of their visual field, but, because of their attentional deficit, they are not aware of them. ${ }^{3}$ So the general picture is this: there is a representation of shape and color, there is a representation of action-properties 
$\underset{\uparrow}{\text { NO Phenomenology (Shape and color) }}$
Representation (Shape and color)

\section{Figure 1}

$\underset{\uparrow}{\text { Phenomenology (Action-properties) }}$
Representation (Action-properties)

$\underset{\uparrow}{\text { NO Phenomenology (Shape and color) }}$
Representation (Shape and color)

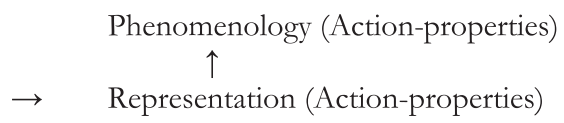

Figure 2

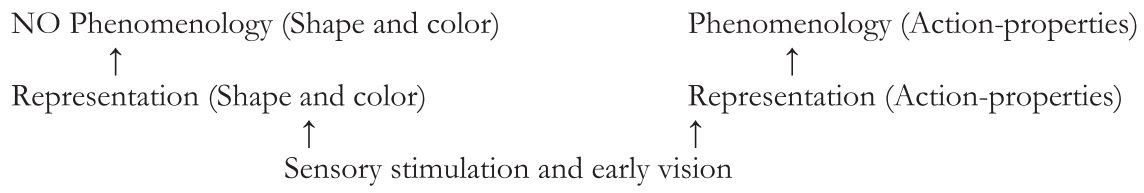

Figure 3

and there is phenomenology of action-properties. But there is no (or there is delayed) phenomenology of shape and color.

This gives us the picture of figure 1 .

One detail that is missing from this picture is the arrow (or lack thereof) between Representation (Shape and color) and Representation (Action-properties).

I'm assuming that it would be absurd to suggest that the representation of shape and color is based on, and grounded in, the representation of actionproperties (in any case, if this were true, we could immediately conclude that action-properties are perceptually represented). This leaves us two options. The first is in figure 2 , and the second in figure 3.

In the case of the first alternative, the representation of shape and color gives rise to the representation of action-properties, which, in turn, is responsible for the phenomenology of action-properties. But for the unilateral neglect patients the phenomenology of shape and color is missing — presumably because the representation of shape and color is not attended to.

The second alternative is that the representation of shape and color and the representation of action-properties are formed independently from one another - our early vision gives rise to both of them independently. According to this account, there are two separate visual subsystems, one responsible for the representation of shape and color and the other responsible for the representation of action-properties. And only the latter, but not the former is accompanied by phenomenology in the unilateral neglect patients.

This second alternative could be thought to be supported by empirical considerations and I need to say a couple of words about these. There is some 
important empirical evidence in favor of the double dissociation between the perceptual subsystem that is responsible for recognition and identification (ventral subsystem) and the one that is responsible for the perceptual guidance of actions (dorsal subsystem) (see Milner and Goodale 1995, Goodale and Milner 2004, Jeannerod 1997). If we apply this empirical evidence to the unilateral neglect case, we might conclude that the ventral subsystem is slow or malfunctioning in unilateral neglect patients, whereas the dorsal subsystem is intact, which is why these patients experience action-properties before (or even without) experiencing its shape or color.

I doubt that relying on the ventral/dorsal distinction is the best bet for the advocates of the second alternative. The double dissociation between the ventral and the dorsal visual subsystems has no immediate implications as to what is visually experienced. Importantly, the distinction is not between the experience of identifying and recognizing and the experience of action control. In fact, the main proponents of the two visual systems hypothesis, Milner and Goodale argue that while ventral processing is typically conscious, dorsal processing is unconscious. This view has been criticized both on empirical and on conceptual grounds (see for example Dehaene et al, 1998, Jeannerod 1997, see also Jacob and Jeannerod 2003, Briscoe 2009, Brogaard forthcoming for summaries): it seems that dorsal processing can also be conscious if it interacts with ventral processing under some special circumstances. There also seems to be evidence that the ventral and the dorsal stream are not as independent from each other as has been previously supposed: they routinely interact at various points of perceptual processing (see, again Jeannerod 1997 for a summary).

What is important for us is not the debate about whether and to what extent dorsal processing interacts with ventral processing and to what extent it can become conscious as a result. What matters from our point of view is that, regardless of whether we accept Milner and Goodale's strict separation of conscious ventral vision and unconscious dorsal vision or Jeannerod's more complex picture where dorsal processing can be made conscious, neither picture gives support to the claim that in the case of the unilateral neglect patients the conscious experience of shape and color is missing (or comes late) because of a breakdown in the ventral stream. The dorsal stream is either unconscious per se (as Milner and Goodale suggest) or can only be made conscious under special circumstances as a result of interaction with ventral processing (as Jeannerod suggests). But neither of these scenarios is consistent with the suggestion that the dorsal stream can give us conscious experience of actionproperties in the complete absence of any conscious experience of shape and color.

These considerations do not show that the first alternative is to be preferred to the second one. All they show is that we should be careful in our attempt at fleshing out the second alternative with the help of the dorsal/ventral distinction. The argument I will present in the next section will apply in the case of both of these alternatives. 


\section{The Argument from Unilateral Neglect}

The argument from unilateral neglect is a reductio argument. The claim that action-properties are not part of perceptual phenomenology yields some very implausible consequences when it comes to characterizing the phenomenal character of the experience of unilateral neglect patients when they are performing the visual search task.

The supposition for reductio is that action-properties are part of the patients' non-perceptual phenomenology. What about their perceptual phenomenology then? The objector is forced to conclude that these patients lack any perceptual phenomenology while they are performing this visual search task. The only properties they are aware of are action-properties, but these properties are, by supposition, not part of their perceptual phenomenology. This is an extremely problematic conclusion as these people are staring at objects, performing visual tasks with what they see, talking about what they see, manipulating what they see, and, importantly, claim to consciously experience what they see. Nonetheless, the objector needs to say that they lack perceptual phenomenology: that there is nothing it is like for them to see these objects.

In other words, the objector is forced to say that it is possible to consciously see an object and nonetheless lack visual phenomenology altogether. If we allow for unconscious perception, it is possible to perceive an object without any accompanying phenomenology, but the consequence of denying that actionproperties are part of perceptual phenomenology is something much more radical: it amounts to saying that it is possible to consciously perceive an object without any accompanying perceptual phenomenology — a claim that comes dangerously close to a straight logical contradiction. If denying that actionproperties are part of perceptual phenomenology forces us to postulate such empty perceptual phenomenology during conscious perception, then we have strong reasons to accept the claim that action-properties are indeed part of perceptual phenomenology.

The aim of the last two sections was to show that action-properties are part of our perceptual phenomenology and thereby providing a case study for the methodology of deciding whether a certain kind of property is part of our perceptual phenomenology. But the main aim of the paper was to provide an unproblematic methodology for deciding what properties are part of our perceptual phenomenology. The case study is supposed to help us to understand what would count as evidence for concluding that a property is part of our perceptual phenomenology. More specifically, we can generalize from the unilateral neglect case study: if we find patients who are capable of experiencing property $\mathrm{P}$ without being capable of experiencing the low-level properties of shape, size and color, then we have good reason to conclude that property $\mathrm{P}$ is part of our perceptual phenomenology. If we want to know which properties are part of our perceptual phenomenology, we need to look for empirical studies of patients with brain lesions. 


\section{Objections}

I need to address some potential objections.

First, some may have a very general worry about my methodology of using findings about a very narrow subset of people, namely, those with unilateral neglect symptoms, in order to arrive at some general claim that would hold for all humans, not only unilateral neglect patients. The answer is simple: if actionproperties were part of non-perceptual phenomenology (for humans in general, healthy or not), then we would get extremely implausible results for unilateral neglect patients.

Second, one could point out that I am not entitled to take action-properties to be part of our perceptual phenomenology without showing that they are not part of our action phenomenology. ${ }^{4}$ It has been argued that action phenomenology is different from, and not reducible to, perceptual phenomenology (see, e.g., Pacherie 2008, Bayne and Levy 2006). Action phenomenology is the phenomenology that accompanies the performance of actions (opinions differ about whether such phenomenology is necessary for the performance of actions and we do not need this extra assumption here).

This proposal would imply that action-properties are part of the unilateral neglect patients' action phenomenology, not their perceptual phenomenology. But then I can run the very same argument I ran against the view that action-properties are part of the unilateral neglect patients' non-perceptual phenomenology. Both of these views lead to the consequence that the unilateral neglect patients' perceptual phenomenology during visual search is empty and I argued above that this consequence is extremely implausible. In short, if my argument against the claim that action-properties are part of our non-perceptual phenomenology is any good, then we can apply it directly against the proposal that action-properties are part of our action phenomenology.

Third, consider the possibility that what happens with the unilateral neglect patients in this visual search experiment is that the unconscious perception of action-properties gives rise directly to the non-perceptual awareness of actionproperties. What rules out this explanation? My answer is that this proposal, just like the proposal about action phenomenology, leads to the implausible consequence that the perceptual phenomenology of these unilateral neglect patients during visual search is empty.

And this leads to the last and probably most important potential objection I need to address. Why is it such an implausible conclusion that the unilateral neglect patients have empty perceptual phenomenology? Couldn't my opponents simply bite the bullet and endorse the claim that these patients' perceptual phenomenology is indeed empty? Because of these considerations, I explicitly avoided saying in the last section that the assumption that action-properties are part of our non-perceptual phenomenology (or the assumption that they are part of our action phenomenology) leads to a straight contradiction. I am not 
sure that it is a straight contradiction to say that the unilateral neglect patients' perceptual phenomenology is empty.

But it comes very close to being a straight contradiction. Let us summarize what we know about the unilateral neglect patients when they are performing the visual search task. First, they have phenomenology: they are aware of actionproperties. And remember that these are action-properties of the very objects that they perceive. Second, they are completing a visual search task: they are performing perceptually-guided actions on the objects that they perceive. If we put these two claims together with the supposition that they lack perceptual phenomenology, what we get is that these subjects consciously experience what they see but have no perceptual phenomenology. Again, not a straight logical contradiction, but a very radical claim indeed.

To demonstrate just how radical this claim is, consider the current debate about cognitive phenomenology. As we shall see in the next section, there is an important debate in philosophy of mind about whether there are conscious non-perceptual states, more specifically, thoughts, that lack any perceptual phenomenology. This divides the philosophical field: some philosophers accept and some deny the existence of conscious non-perceptual states that lack any perceptual phenomenology. But note how much more radical the claim we are considering here is. What we are considering here is not the possibility of conscious non-perceptual states without perceptual phenomenology, but the possibility of conscious perceptual states without perceptual phenomenology. If we want to refrain from embracing this claim, we need to conclude that actionproperties are part of our perceptual phenomenology.

\section{Conclusion: Perceptual versus Non-perceptual Phenomenology}

The aim of this paper was to sketch the right methodology for drawing the distinction between perceptual and non-perceptual phenomenology. I conclude with some remarks on the relevance of this distinction for one of the most important debates in the philosophy of mind.

This debate is about whether all consciousness is perceptual (or quasiperceptual). Some argue that phenomenology is necessarily perceptual phenomenology - that the phenomenal character of non-perceptual states, such as thoughts or beliefs, can be fully accounted for in terms of perceptual phenomenology (Carruthers 2005, Prinz 2007, Robinson 2005, Lormand 1996, Tye 1996, Nelkin 1996). Others deny this and argue that thoughts have distinctive phenomenology that is different from perceptual phenomenology (Pitt 2004, Siewert 1998, Strawson 1994, see also the majority of papers in Bayne and Montague 2011).

It is unlikely that this debate can be settled without having a clear distinction between perceptual and non-perceptual phenomenology. Further, depending on what range of properties one allows to be part of perceptual phenomenology, the plausibility of the two competing views may change. If only shape, size and 
color properties are part of perceptual phenomenology, then the view about the distinctive phenomenology of thoughts may be more tempting, whereas if we allow a wide range of properties to be part of perceptual phenomenology, the view that all consciousness is perceptual may seem more palatable. In other words, we need an unproblematic distinction between perceptual and non-perceptual phenomenology in order to even engage with this debate. The aim of this paper was to sketch a possible methodology for doing so. ${ }^{5}$

\section{Notes}

1. In Nanay forthcoming, I differentiate between thick and thin action-properties. For consistency: what I mean by 'action-property' in this paper is what I defined as 'thick action-properties' in Nanay forthcoming. I will leave off the 'thick' adjective for the sake of simplicity here.

2. The same goes for a variety of unilateral neglect that is known as 'extinction' (Driver and Vuillemmier 2001). Extinction patients can be aware of one stimulus on the contralateral side, but not two (not the one that is further away from the center). The contrast here is between awareness of the first stimulus and the lack of awareness of the second.

3. Note that it is taken for granted in this literature that attention is necessary for consciousness, something I am happy to go along with (see Cohen et al. 2012 for a summary, Prinz 2010 for a philosophical overview, but see also Lamme 2003 for a dissenting view).

4. Thanks to Elisabeth Pacherie for raising this objection.

5. This work was supported by the EU FP7 CIG grant PCIG09-GA-2011-293818 and the FWO Odysseus grant G.0020.12N. I gave earlier versions of this paper at the 2011 Online Consciousness Conference and at the University of Fribourg. I am grateful for my commentators, Kevin Connolly, Farid Masrour and Andrea Giananti as well as comments by Tim Bayne, Carolyn Dicey Jennings, Jordan Dodd, Peter King and Maja Spener.

\section{References}

Bayne, T. 2009. Perception and the reach of phenomenal content. Philosophical Quarterly 59: 385-404.

Bayne, T. \& N. Levy. 2006. The feeling of doing: Deconstructing the phenomenology of agnecy. In Natalie Sebanz \& Wolfgang Prinz (eds.), Disorders of Volition. MIT Press.

Bayne, T. \& M. Montague. 2011. Cognitive Phenomenology. Oxford: Oxford University Press.

Briscoe, R. 2009. Egocentric spatial representation in action and perception. Philosophy and Phenomenological Research 79: 423-460.

Brogaard, B. forthcoming. Conscious vision for action versus unconscious vision for action? Journal of Philosophy.

Carruthers, P. 2005. Conscious experience versus conscious thought. In his Consciousness: Essays from a Higher-Order Perspective. Oxford: Clarendon Press, pp. 134-156.

Cohen, M. A., P. Cavanagh, M. M. Chun \& K. Nakayama. 2012. The attentional requirements of consciousness. Trends in Cognitive Sciences 16: 411-417. 
Dehaene, S., L. Naccache, G. Le Clec'H, E. Koechlin, M. Mueller, G. Dehaene-Lambertz, P.F. van de Moortele \& D. Le Bihan. 1998. Imaging unconscious semantic priming. Nature 395: 597-600.

Driver, J. \& P. Vuilleumier. 2001. Perceptual awareness and its loss in unilateral neglect and extinction. Cognition 79 (1):39-88.

Goodale, M. A. \& A. D. Milner. 2004. Sights Unseen. Oxford: Oxford University Press.

Humphreys, G. W. \& M. J. Riddoch. 2001. Detection by action: Neuropsychological evidence for action-defined templates in search. Nature Neuroscience 4: 84-88.

Jacob, P. \& M. Jeannerod. 2003. Ways of Seeing. The Scope and Limits of Visual Cognition. Oxford: Oxford University Press.

Jeannerod, M. 1997. The Cognitive Neuroscience of Action. Oxford: Blackwell.

Kinsbourne, M. 2006. From unilateral neglect to the brain basis of consciousness. Cortex 42(6): 869-74.

Kriegel, U. 2007. The phenomenologically manifest. Phenomenology and the Cognitive Sciences 6: $115-136$.

Lamme, V.A.F. 2003. Why visual attention and awareness are different. Trends in Cognitive Sciences. 7, 12-18.

Lormand, E. 1996. Nonphenomenal consciousness. Nous 30: 242-261.

Mark, V.W., C.A. Kooistra, \& K. M. Heilman. 1988. Hemispatial neglect affected by nonneglected stimuli. Neurology 38, 1207-1211.

Masrour, F. 2011. Is perceptual phenomenology thin? Philosophy and Phenomenological Research 83: 366-397.

Milner, A. D., \& M. A. Goodale. 1995. The Visual Brain in Action. Oxford: Oxford University Press.

Nanay, B. 2011a. Do we see apples as edible? Pacific Philosophical Quarterly 92: 305-322.

Nanay, B. 2011b. Do we sense modalities with our sense modalities? Ratio 24: 299-310.

Nanay, B. 2012. Action-oriented perception. European Journal of Philosophy, 20: 430-446.

Nanay, B. forthcoming. Between Perception and Action. Oxford: Oxford University Press.

Nelkin, N. 1996. Consciousness and the Origins of Thought. Cambridge: Cambridge University Press.

Pacherie, E. 2008. The phenomenology of action: A conceptual framework. Cognition 107: $179-217$.

Pitt, D. 2004. The phenomenology of cognition. Philosophy and Phenomenological Research 59: $1-36$.

Prinz, J. 2007. All consciousness is perceptual. In B. McLaughlin and J. Cohen (eds.): Contemporary Debates in Philosophy of Mind. Oxford: Blackwell, pp. 335-357.

Prinz, J. 2010. How do perceptual states become conscious? In B. Nanay (ed.): Perceiving the World. New Essays on Perception. New York: Oxford University Press.

Riddoch, M. J., M. G. Edwards, G. W. Humphreys, R. West, \& T. Heafield. 1998. Visual affordances direct action: Neuropsychological evidence from manual interference. Cognitive Neuropsychology 15: 645-693.

Robinson, W. 2005. Thoughts without distinctive non-imagistic phenomenology. Philosophy and Phenomenological Research 70: 534-561.

Schwitzgebel, E. forthcoming Perplexities of Consciousness. Cambridge, MA: MIT Press.

Siegel, S. 2006. Which properties are represented in perception? In T. Gendler \& J. Hawthorne (Eds.), Perceptual Experience. Oxford: Oxford University Press, 481-503.

Siegel, S. 2007. How can we discover the contents of experience? Southern Journal of Philosophy (Supp) 45: 127-142.

Siewert, C. 1998. The Significance of Consciousness. Princeton: Princeton University Press.

Strawson, G. 1994. Mental Reality. Cambridge, MA: The MIT Press.

Treisman, A. 1996. The binding problem. Current Opinions in Neurobiology 6: 171-178.

Tye, M. 1996. Mental reality. Journal of Philosophy 93: 421-424. 